\title{
Mudanças nos atributos quím icos do solo e crescimento de um povoamento de Eucalyptus Grandis fertilizado com composto de resíduos da indústria de celulose e papel
}

\author{
Paulo Fortes Neto ${ }^{1}$; Ana Aparecida da Silva Almeida ${ }^{1}$; Ana Gabriela Monnerat Carvalho \\ Bassa $^{2}$; Claudio Roberto da Silva ${ }^{2}$; Dimas Donizeti Patrocinio ${ }^{2}$ \\ ${ }^{1}$ Universidade de Taubaté - UNITAU \\ Estrada Mun. Dr. José Luiz Cembranelli, 5.000 - 12080-010 - Taubaté - SP, Brasil \\ paulofortes.neto@gmail.com \\ anasilva@unitau.br \\ ${ }^{2}$ FIBRIA \\ Rodovia General Euryale de Jesus Zerbini - 12310-000 - Jacareí - SP, Brasil \\ \{gabriela.monnerat, claudio.silva, dimas.patrocinio\}@fibria.com.br
}

\begin{abstract}
The purpose of this study was to investigate the effect of compost on soil and plant of an compostamended soil under Eucalyptus grandis for one years; from may 2006 to may 2007. Thus, it was evaluated the soil fertility, nutritional status and growth of a Eucalyptus grandis plantation fertilized with compost. The trial was established at the Farm Francos of Guararema - (São Paulo State, Brazil). The experimental design was in complete randomized blocks, with 4 replicates. There were eight treatments, namely: (1) control; (2) mineral fertilization; (3) $15 \mathrm{t} \mathrm{ha}^{-1}$ compost with $100 \%$ dregs; (4) $15 \mathrm{t} \mathrm{ha}^{-1}$ compost with $50 \%$ dregs; (5) $15 \mathrm{t} \mathrm{ha}^{-1}$ compost from aeration passive; (6) $15 \mathrm{t} \mathrm{ha}^{-1}$ compost and inoculation with microorganism; (7) $15 \mathrm{t} \mathrm{ha}^{-1}$ compost with slugdes primary and inoculation with microorganism and (8) $15 \mathrm{t} \mathrm{ha}^{-1}$ compost and mineral fertilization. The applications the compost 360 treatment resulted in a higher soil $\mathrm{Na}$ content and value the PST and C.E. The tratament with mineral fertilization and mineral fertilization more compost proportion higher growth plant.
\end{abstract}

Palavras-chave: compost organic, mineral fertilization, eucalypt, and forest, composto orgânico, fertilização mineral, eucalipto e floresta.

\section{INTRODUÇÃO}

A atividade industrial de celulose e papel atualmente vem desenvolvendo o conceito de reciclar, nas áreas cultivadas com eucalipto, os resíduos orgânicos gerados durante o processamento da celulose e papel. Esta tendência vem ocorrendo porque estes resíduos industriais estão sendo produzidos com menos contaminantes devido às mudanças no processo de fabricação (Harrison, et al., 2003). Vários estudos têm mostrado que o dregs, cinza e o lodo de esgoto, quando aplicados de forma isolada, trazem benefícios agronômicos às culturas - relacionados ao aumento e, ou, manutenção dos níveis de fertilidade do solo em função do fornecimento de nutrientes ou por meio de alterações em seus atributos químicos (Guerrini, et al., 2000; Harrison, et al., 2003; Rocha, et al., 2004; Corrêa et al., 2007; Lira, et al., 2008; Maldonado, 2009). Por outro lado são poucos os estudos para avaliar os benefícios agronômicos do composto orgânico produzido a partir da mistura dos resíduos gerados no processo de produção de celulose e papel.

O presente trabalho teve como objetivo avaliar os efeitos da aplicação do composto produzido com diferentes proporções de resíduos orgânicos nos atributos químicos do solo e no crescimento de um povoamento de Eucalyptus grandis. 


\section{MATERIAL E MÉTODOS}

O estudo foi realizado em uma área de produção comercial de eucalipto localizada no município de Guararema (SP), sobre um Latossolo Vermelho Amarelo distrófico (Embrapa, 1999). O local para realização deste experimento nunca recebeu aplicação de resíduo; e o experimento foi autorizado pelo órgão fiscalizador. No momento da aplicação dos tratamentos, as mudas já estavam estabelecidas na área de estudo há cerca de dois meses. Antes do início do ensaio, foi realizada uma amostragem de solo nas camadas de $0-20 \mathrm{~cm}$ de profundidade.

O composto utilizado foi produzido a partir de uma mistura com diferentes proporções entre lodo biológico, dregs, gritz, biomassa, lodo primário, cinza e resíduo da depuração, gerados durante o processo de produção de celulose e papel.

Os resíduos foram misturados e submetidos à compostagem em pilhas a céu aberto por um período de 120 dias. As pilhas foram montadas e manejadas com as seguintes proporções de resíduos: A- Lodo biológico + dregs + gritz + resíduo da depuração + cinza + casca; B - Lodo biológico + $1 / 2$ geração dregs + gritz + resíduo da depuração + cinza + casca; C - Lodo biológico + dregs + gritz + resíduo da depuração + cinza + casca + inoculação com microrganismos; D - Lodo biológico + lodo primário + dregs + gritz + resíduo da depuração + cinza + casca + inoculação com microrganismos e E - Lodo biológico + dregs + gritz + resíduo da depuração + cinza + casca + com aeração por tubos

As pilhas de A a D foram submetidas ao revolvimento semanal, as pilhas $\mathrm{C}$ e $\mathrm{D}$ foram inoculadas com microrganismos. A pilha $\mathrm{E}$ não foi revolvida e nem inoculada com microrganismos e a aeração ocorreu pela introdução de tubos plásticos perfurados no seu interior.

A composição química e biológica dos compostos orgânicos está apresentada na Tabela 1, os valores de metais pesados e organismos patogênicos ficaram abaixo dos limites estabelecidos pela Resolução CONAMA n ${ }^{\circ} 375$ de 2006, adequando-se para o uso com fins agrícolas.

A dose do composto foi estabelecida em função do conteúdo total de nitrogênio e da quantidade recomendada de nitrogênio para o eucalipto no Estado de São Paulo (120 kg ha ${ }^{-1}$ de N), segundo Raij et al. (1997).

As parcelas experimentais de $378 \mathrm{~m}^{2}$ foram distribuídas em um delineamento experimental de blocos casualizados com quatro blocos e oito tratamentos, conforme apresentado na

\section{Tabela 2.}

O experimento foi iniciado em 16/05/2006 com a aplicação de $15 \mathrm{t} \mathrm{ha}^{-1}$ (base seca) de composto e dos fertilizantes, nos tratamentos correspondentes, na superfície do solo próximo das plantas.

As amostras de terra foram coletadas aleatoriamente nas entrelinhas das parcelas, com o auxílio de um trado introduzido até a camada de $0-20 \mathrm{~cm}$ de profundidade. As amostras foram coletadas antes e após 360 dias da incorporação do composto no solo.

Foram analisados $\mathrm{pH}$ em $\mathrm{CaCl}_{2} 0,01 \mathrm{~mol} \mathrm{~L}^{-1}$, carbono orgânico, fósforo disponível, cálcio, magnésio, potássio, sódio, alumínio trocável, capacidade de troca de cátions, saturação por bases e condutividade elétrica, conforme metodologia preconizada Raij et al. (2001). 
Tabela 1. Alguns atributos dos compostos orgânicos utilizados no cultivo do Eucalyptus grandis. Valores dos elementos e de pH com base na matéria seca

\begin{tabular}{|c|c|c|c|c|c|}
\hline \multirow[t]{2}{*}{ Atributos } & \multicolumn{5}{|c|}{ Composto orgânico } \\
\hline & A & B & C & D & $\mathbf{E}$ \\
\hline $\mathrm{pH}$ & 6,1 & 5,7 & 6,8 & 6,1 & 7,1 \\
\hline Nitrogênio total (\%) & 0,30 & 0,30 & 0,36 & 0,32 & 0,29 \\
\hline Carbono orgânico (\%) & 8,8 & 8,6 & 8,8 & 8,5 & 8,5 \\
\hline Relação C/N & $29 / 1$ & $28 / 1$ & $25 / 1$ & $27 / 1$ & $29 / 1$ \\
\hline Cálcio (\%) & 10,0 & 9,9 & 10,2 & 9,3 & 10,8 \\
\hline Sódio (\%) & 0,48 & 0,60 & 0,36 & 0,44 & 0,45 \\
\hline Magnésio (\%) & 0,83 & 0,81 & 0,69 & 0,78 & 0,88 \\
\hline Potássio (\%) & 0,50 & 0,55 & 0,44 & 0,43 & 0,45 \\
\hline Fósforo (\%) & 0,22 & 0,17 & 0,17 & 0,19 & 0,18 \\
\hline Arsênio $\left(\mathrm{mg} \mathrm{kg}^{-1}\right)$ & $<0,5$ & $<0,5$ & $<0,5$ & $<0,5$ & $<0,5$ \\
\hline Bário $\left(\mathrm{mg} \mathrm{kg}^{-1}\right)$ & 230 & 213 & 276 & 194 & 185 \\
\hline Cádmio (mg kg-1) & $<0,5$ & $<0,5$ & $<0,5$ & $<0,5$ & $<0,5$ \\
\hline Chumbo (mg kg $\left.{ }^{-1}\right)$ & 15 & 9,7 & 14 & 1,9 & 5,5 \\
\hline Cobre $\left(\mathrm{mg} \mathrm{kg}^{-1}\right)$ & 33 & 22 & 39 & 6,4 & 28 \\
\hline Cromo $\left(\mathrm{mg} \mathrm{kg}^{-1}\right)$ & 10,6 & 14 & 19 & 8,5 & 9,9 \\
\hline Mercúrio (mg kg $\left.{ }^{-1}\right)$ & $<0,03$ & $<0,02$ & $<0,03$ & $<0,03$ & $<0,06$ \\
\hline Molibdênio $\left(\mathrm{mg} \mathrm{kg}^{-1}\right)$ & $<0,5$ & $<0,5$ & $<0,5$ & $<0,5$ & $<0,5$ \\
\hline Níquel $\left(\mathrm{mg} \mathrm{kg}^{-1}\right)$ & 1,9 & 32 & 35 & 10 & 35 \\
\hline Selênio $\left(\mathrm{mg} \mathrm{kg}^{-1}\right)$ & $<0,5$ & $<0,5$ & $<0,5$ & $<0,4$ & 0,9 \\
\hline Zinco $\left(\mathrm{mg} \mathrm{kg}^{-1}\right)$ & 12 & 87 & 142 & 27 & 76 \\
\hline Coliformes termotolerantes (NMP g ${ }^{-1}$ de ST) & $<1,8$ & $<1,8$ & $<2,6$ & $<1,5$ & $<1,8$ \\
\hline Ovos viáveis de Helmintos (ovos $\mathrm{g}^{-1}$ de ST) & 0 & 0 & 0 & 0 & 0 \\
\hline Salmonellas em $10 \mathrm{~g}$ de ST & ${ }^{(1)} \mathrm{A}$ & A & A & A & A \\
\hline
\end{tabular}

A determinação da altura das plantas foi realizada 120 dias após o plantio das mudas de eucalipto.

Os resultados foram submetidos à análise da variância com aplicação do teste $\mathrm{F}$ e as médias comparadas entre si pelo teste de Tukey a $5 \%$. 
Tabela 2. T ratamentos aplicados e seus componentes

\begin{tabular}{|c|c|}
\hline Tratamento & Componente \\
\hline T1 & Controle (sem fertilização mineral e aplicação do composto) \\
\hline T2 & $\begin{array}{l}\text { Fertilização M ineral: No plantio: } 110 \mathrm{~g} / \text { planta 04:28:06 + 0,3\% Cu + 0,7\% Zn } \\
\text { Na cobertura: 10 Adubação } 120 \mathrm{~g} / \text { planta 19:00:19 + 4\% S + 0,3B } \\
\text { 20 Adubação } 350 \mathrm{~g} / \text { planta 08:00:36 + 4\% S + 1\% B }\end{array}$ \\
\hline T3 & L odo biológico + dregs + gritz + resíduo da depuração + cinza + casca \\
\hline T4 & $\begin{array}{l}\text { Lodo biológico + 1/2 geração dregs + gritz + resíduo da depuração + cinza + } \\
\text { casca }\end{array}$ \\
\hline T5 & $\begin{array}{l}\text { Lodo biológico }+ \text { dregs }+ \text { gritz }+ \text { resíduo da depuração }+ \text { cinza }+ \text { casca+ } \\
\text { aeração por tubos }\end{array}$ \\
\hline T6 & $\begin{array}{l}\text { Lodo biológico + dregs + gritz + resíduo da depuração + cinza + casca + } \\
\text { inoculação de microrganismos }\end{array}$ \\
\hline T7 & $\begin{array}{l}\text { L odo biológico + lodo primário + dregs + gritz + resíduo da depuração + cinza } \\
\text { + casca + inoculação de microrganismos }\end{array}$ \\
\hline T8 & $\begin{array}{l}\text { Lodo biológico + dregs + gritz + resíduo da depuração + cinza + casca + } \\
\text { Fertilização mineral (igual ao tratamento 02) }\end{array}$ \\
\hline
\end{tabular}

\section{RESULTADO E DISCUSSÃO}

\section{A tributos químicos do solo}

$\mathrm{Na}$ Tabela 3 são apresentados os resultados de pH, P, K, Ca, Mg, M.O e H + Al determinados no solo após 360 dias da aplicação do composto. Observa-se que não foram verificados efeitos significativos dos tratamentos sobre estes elementos químicos no solo. Provavelmente esse resultado está condicionado pela permanência do composto sobre a serapilheira do solo, praticamente sem contato com a biota edáfica responsável pela sua degradação (Chiba, et al., 2008). Nestas condições, os elementos químicos são liberados lentamente na serapilheira e são absorvidos pelas raízes do eucalipto retardando, assim, a sua disponibilidade no solo.

Tabela 3. Atributos de fertilidade do solo na camada de $0-20 \mathrm{~cm}$ de profundidade

\begin{tabular}{|c|c|c|c|c|c|c|c|}
\hline Tratamento & $\mathrm{pH}$ & $\mathbf{P}$ & K & $\mathrm{Ca}$ & $\mathrm{Mg}$ & $\mathrm{H}+\mathrm{AL}$ & M.O \\
\hline & $\mathrm{CaCl}_{2}$ & $\mathrm{mg} \mathrm{dm}^{-3}$ & --------. & $\cdots-\cdots(m$ & $\left.\mathrm{dm}^{-3}\right)$ & --------- & $\mathrm{g} \mathrm{kg}^{-1}$ \\
\hline T1 & 4,0a & $1,0 \mathrm{a}$ & $1,0 \mathrm{a}$ & $2,2 \mathrm{a}$ & $2,7 \mathrm{a}$ & $71,5 \mathrm{a}$ & $27,0 \mathrm{a}$ \\
\hline T2 & $4,1 \mathrm{a}$ & $1,0 \mathrm{a}$ & $1,1 \mathrm{a}$ & $3,2 \mathrm{a}$ & $2,5 \mathrm{a}$ & $69,5 \mathrm{a}$ & $24,0 \mathrm{a}$ \\
\hline T3 & $4,2 \mathrm{a}$ & $3,0 \mathrm{a}$ & $1,1 \mathrm{a}$ & $11,7 \mathrm{a}$ & $2,5 \mathrm{a}$ & $67,0 \mathrm{a}$ & $24,0 \mathrm{a}$ \\
\hline T4 & 4,0a & $7,0 \mathrm{a}$ & $1,2 \mathrm{a}$ & $7,5 \mathrm{a}$ & $3,5 \mathrm{a}$ & $57,7 \mathrm{a}$ & $27,0 \mathrm{a}$ \\
\hline T5 & $4,6 \mathrm{a}$ & $14,0 \mathrm{a}$ & $1,2 \mathrm{a}$ & $13,2 \mathrm{a}$ & $3,0 \mathrm{a}$ & $56,2 \mathrm{a}$ & $31,0 \mathrm{a}$ \\
\hline T6 & $4,2 \mathrm{a}$ & $9,0 \mathrm{a}$ & $1,2 \mathrm{a}$ & $13,5 \mathrm{a}$ & $2,7 \mathrm{a}$ & $53,5 \mathrm{a}$ & $28,0 \mathrm{a}$ \\
\hline T7 & $4,2 \mathrm{a}$ & $3,0 \mathrm{a}$ & $1,3 \mathrm{a}$ & $7,25 \mathrm{a}$ & $2,5 \mathrm{a}$ & $52,7 \mathrm{a}$ & $25,0 \mathrm{a}$ \\
\hline T8 & $4,5 \mathrm{a}$ & $4,0 \mathrm{a}$ & $1,0 \mathrm{a}$ & $10,0 \mathrm{a}$ & $3,5 \mathrm{a}$ & $48,2 \mathrm{a}$ & $28,0 \mathrm{a}$ \\
\hline
\end{tabular}

Médias seguidas de mesma letra, na coluna, não diferem entre si pelo teste de Tukey $(\mathrm{p}<0,05)$. 
Os resultados de sódio, potencial de sódio trocável (PST) e condutividade elétrica do solo estão apresentados na Figura $\mathbf{1}$ observa-se um incremento significativo no teor de sódio e nos valores do PST e C.E nas parcelas com aplicação do composto.

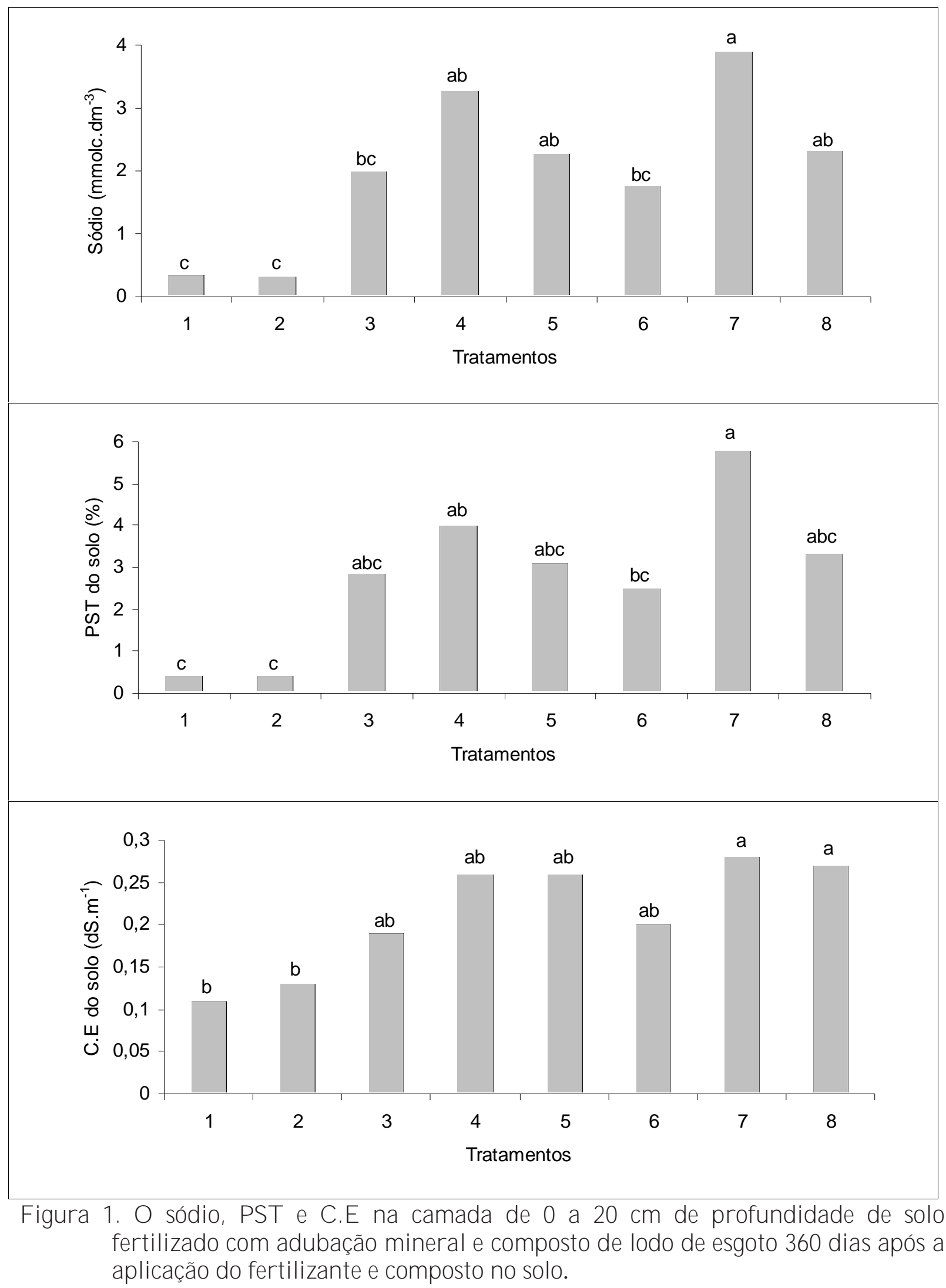


Os valores de sódio variam de $0,3 \mathrm{mmol}_{\mathrm{c}} \cdot \mathrm{dm}^{-3}$ nos tratamentos sem aplicação do composto para $3,9 \mathrm{mmol}_{\mathrm{c}} \cdot \mathrm{dm}^{-3}$ no tratamento $7 \mathrm{com}$ a adição do composto. O PST segue a mesma tendência do sódio com os valores variando de $0,4 \%$ nos tratamentos sem adição do composto para 5,8\% no solo com a aplicação do composto do tratamento 7. Apesar do aumento do PST, verifica-se que os valores determinados nos tratamentos com a adição do composto não ultrapassaram o valor de $7 \%$, considerado como limite para evitar o risco de dispersão de argila no solo (Pizarro, 1978).

A condutividade elétrica aumentou em função da adição do composto aplicado ao solo, sendo mais significativo nos tratamentos 7 e 8 (Figura 1); apesar do aumento dos valores, eles ficaram abaixo do limite de $2 \mathrm{dS} / \mathrm{m}$, considerado como de risco de salinização para o solo (Glossary of Soil Science Terms, 1997).

\section{Desenvolvimento das plantas}

A fertilização mineral isolada (T2) e combinada com o composto orgânico (T8) influenciou significativamente a altura e o DAP das plantas aos 360 dias após o plantio das mudas Figuras 2. Esses resultados sugerem que o uso do composto deverá estar associado a uma suplementação com fertilizante mineral.

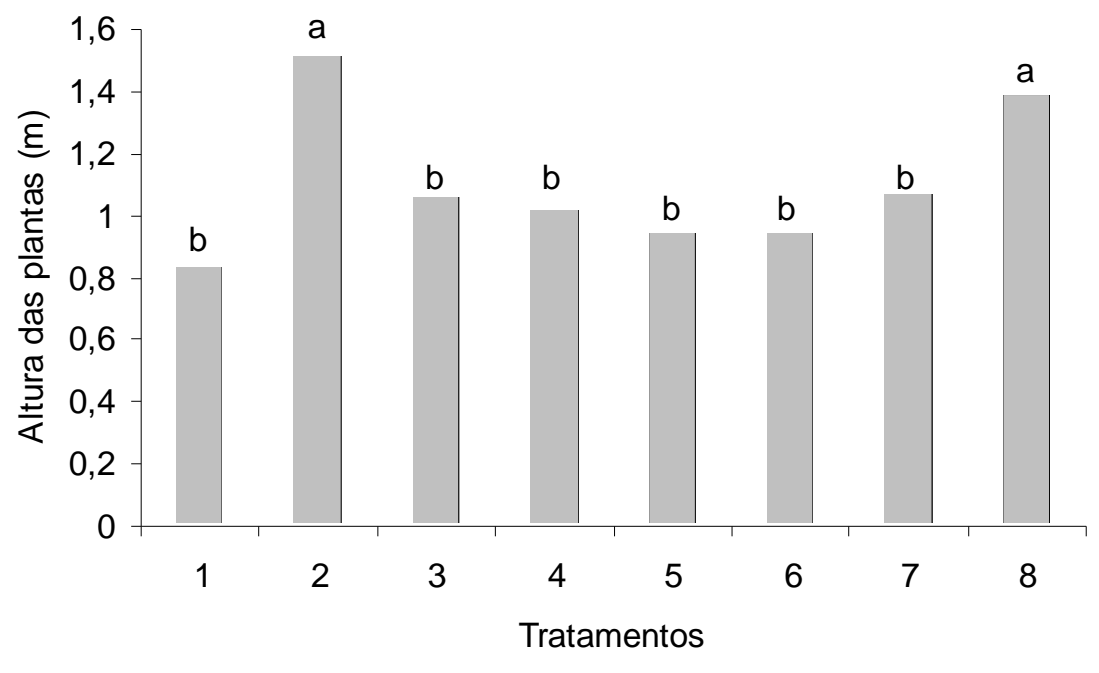

Figura 2. A altura e o DAP de Eucalipytus grandis fertilizado com adubação mineral e composto de lodo de esgoto determinados aos 360 dias após o plantio.

\section{CONCLUSÕES}

- A aplicação do composto não causou efeitos deletérios na qualidade do solo, mesmo em termos de aporte de sódio

- A aplicação do composto suplementado com fertilização mineral favoreceu o desenvolvimento das plantas.

- A aplicação do composto de lodo no solo resultou em aumento nos valores do PST e C.E, porém em níveis abaixo dos valores de risos de salinização. 


\section{LITERATURA CITADA}

Chiba, M.K.; Mattiazzo, M.E. \& Oliveira, F.C. cultivo de cana-de-açúcar em argissolo tratado com lodo de esgoto. II - fertilidade do solo e nutrição da planta. Revista Brasileira da Ciência do Solo, 32:653-662, 2008.

CONSELHO NACIONAL DO MEIO AMBIENTE - CONAMA. Resolução n ${ }^{\circ}$ 375/06. Define critérios e procedimentos, para o uso agrícola de lodos de esgoto gerados em estações de tratamento de esgoto sanitários e seus produtos, e dá outras providencias. Disponível em: http://www.mma.gov.br/port/conama/res/res06/res37506.pdf. Acesso em: 01 jan 2007.

Corrêa, J.C.; Büll, L.T.; Crusciol, C.A.C.; Marcelino, R.; Mauad, M. Correção da acidez e mobilidade de íons em Latossolo com aplicação superficial de escória, lama cal, lodos de esgoto e calcário. Pesquisa Agropecuária Brasileira, v.42, n.9, p.1307-1317, 2007.

EMPRESA BRASILEIRA DE PESQUISA AGROPECUÁRIA - EMBRAPA. Centro Nacional de Pesquisa de Solos. Sistema brasileiro de classificação de solos. Rio de Janeiro, 1999. 412p.

Guerrini, I.A.; Villas Bôas, L.I.; Benedetti, V.; Comério, J.; Moro, L. Application of wood ash and pulp and paper sludge to Eucalyptus grandis in three Brazilian soils. In: Henry, C.L.; Harrison, R.B.; Bastian, R.K., ed. The For esty Alternative:principles and practice of residual use. Seattle: College of Forest Resources, University of Washington, 2000, p 127-131.

Glossary of Soil Science Terms. Madison, WI: Soil Science Society of America, 1997. 134p.

Harrison, R.B; Guerrini, I.A.; Henry, C.L.; Cole, D.W. Reciclagem de residuos industriais e urbanos em áreas de reflorestamento. Piracicaba: Instituto de Pesquisas e Estudos Florestais, 2003. 23p.

Lira, A.C.S.; GUEDES, M.C.; SCHALCH, V. Reciclagem de lodo de esgoto em plantação de eucalipto: carbono e nitrogêno. Engenharia Sanitária e A mbiental, Rio de Janeiro, v.13, n.2, p.207-216, 2008.

Maldonado, C.A.B. Fertilidade do solo, nutrição de plantas de eucalipto ao longo de 46 meses após aplicação de lodo de esgoto. 2009. 116 p. Tese (Doutorado em Energia Nuclear na Agricultura e no Ambiente) - Universidade de São Paulo, Piracicaba, 2009.

Pizarro, F. Drenaje agrícola y recuperación de suelos salinos. 1ed. Madrid: Editora Agrícola Española S.A, 1978. 521p

Raij, B.van; Andrade, J.C.; Cantarella, H. \& Quaggio, J.A. Análise química para avaliação da fertilidade de solos tropicais. Campinas, Instituto Agronômico, 2001. 285p.

Raij, B.van; Cantarella, H.; Quaggio, J.A. \& Furlani, A.M.C., eds. Cana-de-açúcar. In: Recomendações de adubação e calagem para o E stado de São Paulo. 2.ed. Campinas, Instituto Agronômico - Fundação IAC, 1997. p.237-239. (Boletim Técnico, 100).

Rocha, G.N.; Gonçalves, J.L.M.; Moura, I.M. Mudanças da fertilidade do solo e crescimento de um povoamento de Eucalyptus grandis fertilizado com biossólido. Revista Brasileira da Ciência do Solo, 28:623-639, 2004. 
Anais II Seminário de Recursos Hídricos da Bacia Hidrográfica do Paraíba do Sul: Recuperação de Áreas Degradadas, Serviços Ambientais e Sustentabilidade, Taubaté, Brasil, 09-11 dezembro 2009, IPABHi, p. 289-296.

(doi:10.4136/serhidro.37) 